\title{
DESEMPENHO DE HÍBRIDOS DE MILHO TIPO AMILÁCEO EM RELAÇÃO A CARACTERÍSTICAS DO RENDIMENTO DE GRÃOS
}

\author{
AMALIO MENDOZA ${ }^{1}$, COSME DAMIÃO CRUZ ${ }^{2}$ e VERONICA MACHADO
}

\author{
${ }^{1}$ Técnico do Programa de Investigação Milho, Sorgo e Girassol (PIMSG). \\ Instituto Paraguaio de Tecnologia Agrária (IPTA - CECH). Choré, Paraguai-amalio.mendoza@ipta.gov.py. \\ ${ }^{2}$ Departamento de Biologia Geral, Centro de Ciências Biológicas e da Saúde, \\ Universidade Federal de Viçosa, Viçosa, Minas Gerais, Brasil-cdcruz@ufv.br. \\ ${ }^{3}$ Fitomelhoradora Aposentada do Programa de Investigação de Milho, Sorgo y Girassol (PIMSG). \\ Instituto Paraguaio de Tecnologia_Agrária(IPTA).RuaVI, Km 16, Capitán Miranda,Paraguai-verónica_machadocorrea@hotmail.
}

Revista Brasileira de Milho e Sorgo, v.18, n.1, p. 74-87, 2019

\begin{abstract}
RESUMO - O objetivo deste trabalho foi avaliar a existência de interação entre genótipos e ambientes e estimar os parâmetros de adaptabilidade e estabilidade de genótipos de milho amiláceo. Para isto, 44 genótipos de milho amiláceo de três ambientes das localidades da Região Oriental do Paraguai foram utilizados para estimar os parâmetros de adaptabilidade e estabilidade para quatro características. As características usadas foram floração masculina, altura de planta até o ápice da panícula, aspecto da planta e rendimento. O delineamento experimental utilizado foi o de blocos ao acaso, com três repetições e parcelas contendo 25 plantas. $\mathrm{O}$ teste $\mathrm{F}$ demonstrou diferenças significativas para todas as características avaliadas na localidade de Choré com nível de 1\%. Para a localidade de Capitán Miranda, no entanto, produtividade de grãos (PG) e aspecto da planta (AP) não foram significativos. Na localidade Yjhovy, o (PG) foi significativo aos 5\%. A análise de variância conjunta mostrou uma interação significativa para genótipos por ambientes ao nível de 5\% de significância pelo teste F, para todas as características, com exceção de floração masculina. A maioria das características apresentou interação do tipo complexa. Os materiais 41 e 24 foram os genótipos que contiveram os menores valores de índice de superioridade do cultivar (Pi) geral.
\end{abstract}

Palavras chave: Adaptabilidade e estabilidade, interação genótipo ambiente, Avati Morotî, raças.

\section{PERFORMANCE OF AMYLACE TYPE CORN HYBRIDS IN RELATION TO GRAIN YIELD CHARACTERISTICS}

\begin{abstract}
In this study, the aim was to evaluate the interactions between genotypes and environments, and estimate the parameters of adaptability and stability of genotypes of maize starch. Forty four genotypes of maize starch from three environments of the locations of the Eastern Region of Paraguay were used to estimate the parameters of adaptability and stability for four features. The features used were the male flowering, height to the panicle apex, plant aspect and yield. The experimental design was a randomized block design with three replications, each containing 25 plants. The $\mathrm{F}$ test showed significant differences for all traits in the locality of Chore with level of $1 \%$, while the location of Captain Miranda grain yield (GY) and plant aspect (AP) were not significant. In the locality Yjhovy, the (PG) was significant at $5 \%$. The analysis of variance showed a significant interaction for genotype by environment at the $5 \%$ significance level for the F test, for all traits except male flowering. Most features of the showed interaction of complex type. The materials were 41e 24 genotypes that contained the lowest values of index of superiority do cultivar (Pi) general.
\end{abstract} Keywords: Adaptability and stability, Avati Morotî, breeds, genotype environment interaction. 
O milho amiláceo é um dos principais alimentos dos habitantes rurais do Paraguai. A produção é principalmente destinada ao consumo em forma de milho verde, grãos e fubá, principal componente dos diferentes pratos típico do país.

Os produtores do milho amiláceo em sua maioria são pequenos agricultores com baixos níveis de instrução, escassa capacidade empresarial e limitado poder de negociação na cadeia produtiva do milho. $\mathrm{O}$ minifúndio é um fator que incide fortemente na falta de produção em maior escala; porém, a produção do milho para consumo, na sua forma verde ou como fubá, é a mais importante fonte de ingresso para os produtores deste tipo de milho no país.

$\mathrm{O}$ rendimento nacional proporcionado pelo pequeno produtor está entre 1.062 e $2.500 \mathrm{~kg} \mathrm{ha}^{-1}$ (Censo, 2009 citado por González, 2014). Isto se deve a fatores, tais como uso de sementes de baixa qualidade, manejo inadequado e variedades de baixo valor genético com ciclo longo e produtividade baixa. Frente a esta situação, o IPTA (Instituto Paraguaio de Tecnologia Agrária), em parceria com INBIO (Instituto de Biotecnologia Agrícola), vem trabalhando para gerar variedades de maior produtividade e ciclo precoce para o pequeno e médio produtor.

Normalmente as variedades (nativas) utilizadas pelos pequenos produtores têm pouco processo de melhoramento ou foram introduzidas de outras localidades ou de outros países sem um estudo de adaptabilidade e estabilidade. Trabalho realizados por Machado et al. (2002) e Machado (2013) mostraram importância do estudo de adaptabilidade e estabilidade para milhos antes de serem liberados no Paraguai.

Uma etapa importante no programa de melhoramento da espécie refere-se à recomendação de cultivares. Entretanto, ao se testar genótipos em diferentes ambientes poderá ocorrer interação entre os genó- tipos e os ambientes, de forma que um genótipo superior, em um determinado ambiente, poderá não ser em outro ambiente, dificultando sua recomendação. A interação genótipo por ambiente (GxA) tem inúmeras implicações para os programas de melhoramento. Em milho, ela influencia especialmente a etapa de avaliação de híbridos superiores para a indicação de cultivares. Assim, a avaliação de híbridos precisa ser conduzida em diferentes condições climáticas, edáficas e de manejo, o que torna essa etapa uma das mais trabalhosas e onerosas (Ribeiro \& Almeida, 2011). Quando a interação GxA apresenta-se significativa, três estratégias podem ser adotadas para atenuar o seu efeito: i) identificar cultivares específicas para cada ambiente, o que geralmente é inviável em razão do custo; ii) identificar genótipos com baixos níveis de interação, sendo a estratificação de ambientes em sub -regiões mais homogêneas uma estratégia que facilita a seleção de genótipos; e iii) identificar cultivares com maior estabilidade fenotípica (Eberhart \& Russel, 1966). Quando se dispõe de uma rede de ambientes para avaliação das cultivares, é fundamental avaliar ainda o grau de representatividade dos ensaios da faixa de adaptação da cultura, pois permite tomar decisões com relação a descartes de ambientes quando existirem problemas técnicos ou escassez de recursos. Além disso, permite identificar grupos de ambientes em que os genótipos apresentam padrões similares de resposta às variações ambientais, visando maior sucesso na recomendação deles (Cruz et al., 2012).

A interação GxA pode ser dividida em duas partes. Uma de natureza simples, quando não ocorre alteração das posições relativas dos genótipos avaliados, dentro de um conjunto de ambientes, tomados dois a dois. A outra, chamada de complexa, ocorre quando a correlação entre o desempenho dos genótipos ao longo dos ambientes em estudo é baixa, o 
que faz com que a posição relativa dos genótipos seja alterada em virtude das diferentes respostas às variações ambientais (Robertson, 1959).

Este comportamento diferencial atribuído à interação GxA e sua existência exige do melhorista estudo detalhado do comportamento das cultivares, por meio da análise de adaptabilidade e estabilidade, para garantir maior segurança às indicações de cultivares. Existem vários métodos para a avaliação da adaptabilidade e estabilidade de genótipos, que são complementares à análise individual e conjunta dos dados experimentais resultantes de ensaios realizados em uma série de ambientes (Cruz et al., 2012). Esses métodos devem ser empregados quando ocorre interação GxA.

Visando a recomendação de cultivares de milho para a região Oriental do Paraguai, o IPTA usa redes de ensaios distribuídas em vários campos e centros de investigação do país. No presente trabalho foram estudados três locais contrastantes e localizados em regiões com alta produção de milho, com o intuito de avaliar o comportamento dos híbridos, a interação GxA, o tipo de interação e o híbrido mais adaptado e estável para uma recomendação ao produtor.

\section{Material e Métodos}

O estudo foi realizado em três localidades da região Oriental do Paraguai, Capitan Miranda - Estado de Itapúa, situado a $27^{\circ} 13$ ' 12 " S e $55^{\circ} 47^{\prime \prime} 59^{\prime}$ W com 200 msnm; Choré -Estado de San Pedro, situado a $24^{\circ} 11^{\prime \prime} 60^{\prime} \mathrm{S}$ e $56^{\circ} 34^{\prime \prime} 00^{\prime} \mathrm{W}$ com $250 \mathrm{msnm}$ e Yjhovy - Estado de Canindeyú, situado a 2415' S e 55 60'W com 200 msnm. Esses locais são considerados ambientes contrastantes em relação a condições de solo, López et al. (1995), e de clima, Paraguai (2014).
As precipitações médias durante o período detrabalho nas três localidades podem ser observadas na Figura 1.

Foram utilizados 70 cruzamentos simples de diferentes origens, semeados em duas fileiras de dez metros de comprimento, com separação de 0,90 m entre fileiras e 0,20 m entre plantas. Com base na informação sobre o ciclo dos cruzamentos simples, foram selecionados quatro grupos de maturação e cada um deles foi semeado em forma escalonada permitindo o maior número de cruzamentos possível.

As polinizações foram realizadas entre os dias 02/02/2011 e 24/02/2011, obtendo-se um total de 145 híbridos duplos. Foram selecionados 132 híbridos duplos com suficientes sementes para serem avaliados durante dois anos e em três localidades diferentes.

Para facilitar o manejo dos ensaios, os 132 cruzamentos foram divididos em três grupos de 44 híbridos cada um, de acordo com a precocidade, sendo o Grupo I, tardio, o Grupo II, Intermediário e Grupo III, Precoce. A cada grupo foram agregadas cinco cultivares como testemunhas: a variedade experimental GVS 254; duas variedades do Programa de Investigação de Milho (PIM-IPTA), GV 252 e GV 253; e duas variedades comumente utilizadas pelos agricultores, AM Choré e AM San Juan Bautista. Neste trabalho só foi considerado o grupo Precoce.

Foi considerado o delineamento de blocos casualizados com três repetições. O tamanho da unidade experimental foi uma fileira de 5,00 metros de comprimento, com separação de 0,90 metros entre fileiras e 0,20 metro entre plantas, ajustando assim uma população de 55.555 plantas por hectares.

Foram avaliadas as características: Floração masculina (FM): data em que $50 \%$ das plantas na parcela útil emitem pólen, expressa em dias; Altura de planta até o ápice da panícula (APl): média de 
Tabela 1. Resultados das análises de solo das localidades da pesquisa. Paraguai. Biênio 2011/12.

\begin{tabular}{|c|c|c|c|c|c|c|c|c|c|c|c|c|}
\hline \multirow{2}{*}{ Localidades } & \multirow{2}{*}{$\begin{array}{c}\mathbf{p H} \\
\left(\mathbf{H}_{2} \mathbf{O}\right)\end{array}$} & \multirow{2}{*}{$\begin{array}{c}\% \\
\text { М.O. }\end{array}$} & \multicolumn{5}{|c|}{$\mathrm{cmol}^{+} / \mathbf{L ~ S}$. } & \multicolumn{5}{|c|}{$\mathrm{mg} / \mathrm{L} \mathrm{S}$. } \\
\hline & & & $\mathrm{Al}^{+3}$ & $\mathrm{Ca}^{+2}$ & $\mathrm{Mg}^{+2}$ & $\mathbf{K}^{+}$ & $\mathbf{P}$ & $\mathbf{C u}$ & $\mathrm{Fe}$ & Mn & Zn & $\mathbf{N a}$ \\
\hline Choré & 6,2 & 1,34 & 0,00 & 2,60 & 1,11 & 0,56 & 17,37 & 0,00 & 0,00 & 0,00 & 0,00 & 0,00 \\
\hline Yjhovy & 5,6 & 3,82 & 0,00 & 13,00 & 2,99 & 1,04 & 14,69 & 0,00 & 0,00 & 0,00 & 0,00 & 0,00 \\
\hline Cap.Miranda & 5,9 & 3,28 & 0,00 & 20,20 & 3,82 & 0,80 & 38,74 & 0,00 & 0,00 & 0,00 & 0,00 & 0,00 \\
\hline
\end{tabular}

As precipitações médias durante o período detrabalho nas três localidades podem ser observadas na Figura 1.

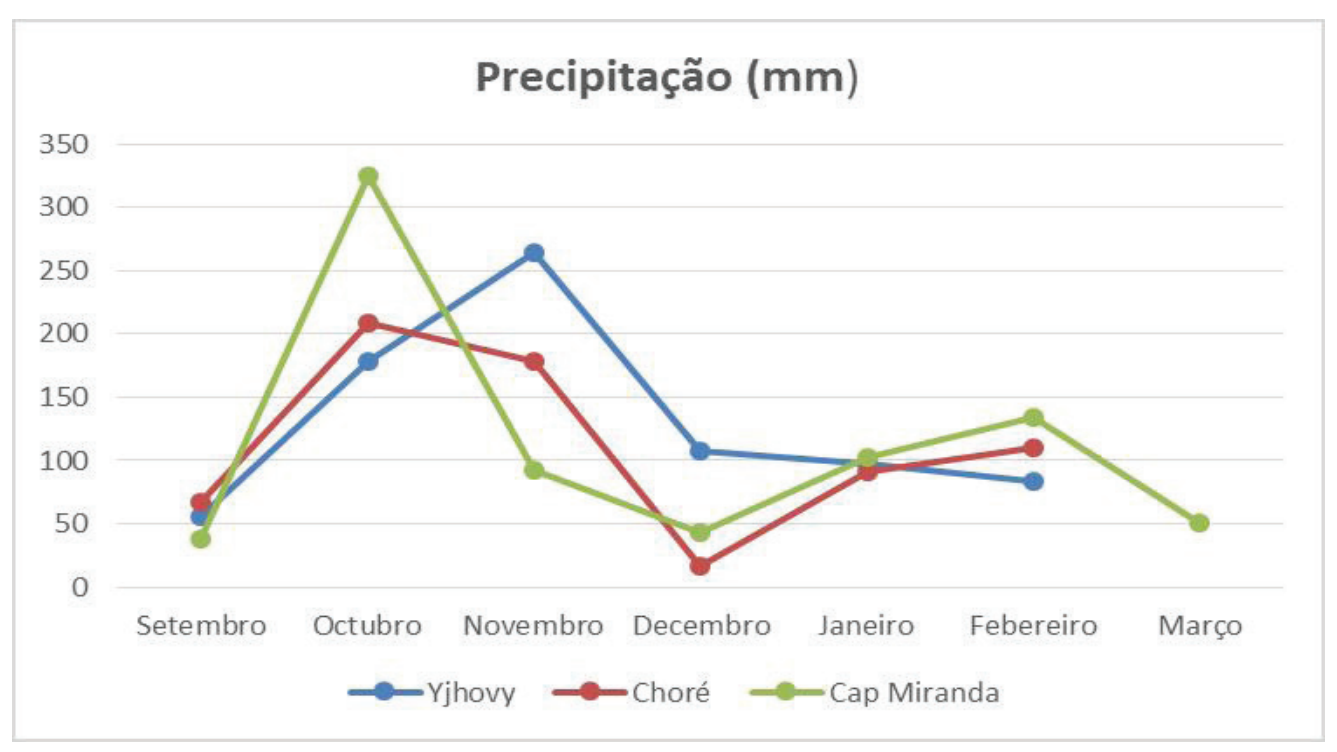

Figura 1. Precipitações durante o período experimental nas três localidades. Paraguai. Biênio 2011/12.

três plantas, medida desde o nível do solo até o ápice da planta, realizada em algum momento desde o início da maturação fisiológica até o dia da colheita, expressa em centímetros; Aspecto de planta (AP): registrada com as plantas ainda verdes e com espigas totalmente formadas, qualificando-se pela uniformidade da altura de inserção das espigas e da planta; danos causados por insetos e doenças, plantas tombadas - quebradas em especial ao rendimento esperado de acordo ao tamanho e número de espigas na planta. Utilizou-se escala de 1 a 5, em que 1 é considerada melhor condição e 5 piores condições; e Rendimen- to (PG): calculado usando o peso de grão obtido na parcela útil e a área da mesma parcela, expresso em quilogramas por hectares.

O rendimento e o peso de 1.000 grãos foram ajustados a $13 \%$ de umidade. Para calcular o rendimento por hectare efetuou-se uma correlação por população dentro de cada unidade experimental pela fórmula adaptada de Leclerg (1966):

$$
\text { Pcorregido }=\text { Pcampo }\lfloor(42)-(0,3 * M) /(42-M)\rfloor
$$

em que:

$M=$ número de plantas faltantes 
E para a correção da umidade foi usado o método proposto por Avila e Sanches (1978)

Pcorregido $=$ Pcampo $\lfloor(100)-(\% U) /(100-13)\rfloor$

em que:

$\mathrm{U}=$ umidade de grão.

Os dados foram submetidos à análise de variância, e as médias foram comparadas pelo procedimento de agrupamento de Scott-Knott (Scott \& Knott, 1974), em nível de 5\% de probabilidade.

Inicialmente, os dados de cada ambiente foram submetidos à análise de variância individual para todos os ambientes, visando detectar variabilidade entre os híbridos e a testemunhas, com base no modelo:

$Y i k=\mathrm{m}+G i+B k+E i k$

em que:

$Y i k=$ valor observado do i-ésimo genótipo, no k-ésimo bloco;

$\mathrm{m}=$ média geral;

$G i=$ efeito do i-ésimo Tratamento T [genótipo i $(\mathrm{i}=1,2, \ldots, \mathrm{g})$ testemunha $\mathrm{t}(\mathrm{t}=1,2 \ldots, \mathrm{t})]$;

$B k=$ efeito do bloco $\mathrm{k}(\mathrm{k}=1,2, \ldots, \mathrm{r})$; e

$E i k=$ erro aleatório.

A análise conjunta foi realizada visando estimar o componente da interação genótipos x ambientes, conforme o modelo a seguir:

Yijk $=\mathrm{m}+\mathrm{Gi}+\mathrm{B} / \mathrm{Ajk}+\mathrm{Aj}+\mathrm{GAij}+\mathrm{Eijk}$

Yijk = valor observado do i-ésimo genótipo, no j-ésimo ambiente e no k-ésimo bloco;

$m=$ média geral;

$G i=$ efeito do i-ésimo Tratamento (Fixo) $\mathrm{T}$ [genótipo i $(\mathrm{i}=1,2, \ldots, \mathrm{g})$ testemunha $\mathrm{t}(\mathrm{t}=1,2 \ldots, \mathrm{t})$ ];

$\mathrm{B} / \mathrm{Ajk}=$ efeito do bloco $\mathrm{k}$ dentro do ambiente $\operatorname{Aj}(k=1,2, \ldots, r)$;

$\mathbf{A j}=$ efeito do $\mathrm{j}$-ésimo ambiente, $(\mathrm{j}=1,2, \ldots, \mathrm{a})$;
GAij = efeito da interação do i-ésimo genótipo com o j-ésimo ambiente; e

Eijk = erro médio aleatório.

A decomposição da interação em parte complexa foi realizada segundo Cruz e Castoldi (1991). Essa decomposição estabelece que a parte complexa seja obtida pela expressão: $C=\sqrt{(1-r)^{3}} \sqrt{Q_{1} \cdot Q_{2}}$, sendo $Q_{1}$ e $Q_{2}$ os quadrados médios dos genótipos nos ambientes 1 e 2, respectivamente, e $r r$ a correlação entre as médias dos genótipos nos dois ambientes.

Com os resultados, realizou-se posteriormente a análise de adaptabilidade e estabilidade fundamentada em estatística não paramétrica de Lin e Binns (1988), caracterizada pela expressão:

$$
P i=\sum_{j=1}^{\mathrm{a}}\left(\frac{\mathrm{X}_{\mathrm{ij}}-\mathrm{M}_{\mathrm{j}}}{2 \mathrm{a}}\right)^{2}
$$

Em que $\boldsymbol{P} \boldsymbol{i}$ é o índice de superioridade da cultivar, $\mathbf{X}_{\mathbf{i j}}$ representa o rendimento da cultivar, onde $\boldsymbol{j}$ é o ambiente, $\boldsymbol{M}$ é a máxima resposta observada entre todas as cultivares no ambiente a e a o número dos ambientes.

Essa expressão foi desdobrada em:

$$
P_{i}=\frac{\left[a\left(X_{i .}-\bar{M}\right)^{2}+\sum_{j=1}^{a}\left(X_{i j}-\bar{X}_{i .}-M_{j}+\bar{M}\right)^{2}\right]}{2 a}
$$

em que:

$$
\begin{aligned}
Y_{i .} & =\sum_{j=1}^{a} Y_{i j} / a: \text { média do híbrido duplo } \\
\bar{M} & =\sum_{j=1}^{a} M_{j} / a: \text { média do hibrido duplo com resposta máxima }
\end{aligned}
$$

Considerando que $M_{j}$ representa uma cultivar hipotética, então o primeiro termo da equação representa a soma de quadrados para efeito genético e o segundo termo é a soma de quadrados para o efeito da interação genótipos $\mathrm{x}$ ambientes quando duas cultivares são comparadas, sendo o segundo o mais importante. 
As análises estatísticas foram realizadas utilizando o Programa Genes (Cruz, 2013).

\section{Resultados e Discussão}

Na Tabela 2, são apresentados os resumos das análises de variância em cada ambiente, a média e o coeficiente de variação experimental $(\mathrm{CV})$ para os caracteres avaliados. As análises evidenciaram boa precisão experimental, avaliada pelo CV (Scapim et al., 1995), com estimativas inferiores a $18,12 \%$ para produtividade de grãos.

Considerando a produtividade de grãos, observou-se que, nas localidades de Choré e Yjhovy, houve diferença significativa $(\mathrm{P}<0,05)$ entre os híbridos. $\mathrm{O}$ ensaio de Capitán Miranda não apresentou diferenças significativas para produtividade de grãos. A ausência de diferenças significativas entre os híbridos no ensaio de Capitán Miranda pode ter sido ocasionada em razão do estresse hídrico (seca), registrada nessa lo- calidade, de forma que os híbridos apresentaram baixas produtividades (Figura 1). Os ensaios de Yjhovy apresentaram boas condições ambientais, principalmente de solo, por se constituir de um campo experimental recém-implantado, para o desenvolvimento dos híbridos. Em relação ao contraste Híb. vs Test, foram registradas diferenças, com alta significância $(\mathrm{P}<0,01)$, para as localidades de Capitán Miranda e Choré. Não foi detectada significância estatística para o contraste em Yjhovy, isto poderia ser pelo ensaio ter sido instalado em parcela de pouco uso, com boa fertilidade, permitindo assim desenvolvimento homogêneo para o tratamento (Tabela $\mathbf{1}$ ).

Para a variável aspecto de planta (AP), observou-se que, nas localidades de Choré e Yjhovy, houve diferença significativa $(\mathrm{P}<0,01)$ entre os híbridos. O ensaio de Capitán Miranda foi novamente o que não apresentou diferenças significativas; essa falta de significância pode ter sido pelas condições desfavoráveis para este local. Portanto, o contraste

Tabela 2. Quadrados médios, médias e coeficientes de variação $(\mathrm{CV})$ para produtividade de grãos em $\mathrm{kg} \mathrm{ha}^{-1}$ (PG), aspecto de planta (AP), floração masculina em dias (FM) e altura de planta em cm (APl) avaliados em três ensaios de milho amiláceo. Paraguai. Biênio 2011/12.

\begin{tabular}{|c|c|c|c|c|c|c|c|c|c|c|c|c|c|}
\hline \multirow{3}{*}{ FV } & \multicolumn{5}{|c|}{ CAPITAN MIRANDA } & \multicolumn{4}{|c|}{ CHORE } & \multicolumn{4}{|c|}{ YJHOVY } \\
\hline & \multirow[t]{2}{*}{ GL } & \multicolumn{4}{|c|}{ QM } & \multicolumn{4}{|c|}{ QM } & \multicolumn{4}{|c|}{ QM } \\
\hline & & PG & $\mathbf{A P}$ & FM & API & PG & $\mathbf{A P}$ & FM & API & PG & $\mathbf{A P}$ & FM & API \\
\hline BLOCO & 2 & 773969.33 & 0,10 & 2,59 & 1200,67 & 1215971,43 & 0,15 & 2,59 & 1881,77 & 1595309,41 & 1,48 & 0,12 & 589,89 \\
\hline TRAT & 48 & $413537,45^{* *}$ & $0,62 * *$ & $46,85^{* *}$ & $211,81 * *$ & $879300,84 * *$ & $0,46 * *$ & $41,84 * *$ & $432,58 * *$ & $1793461,01^{*}$ & $0,57 * *$ & $24,97 * *$ & $534,00 * *$ \\
\hline HIB & 43 & $273609,97 \mathrm{~ns}$ & $0,41 \mathrm{~ns}$ & $27,37 * *$ & $200,56 * *$ & $833596,28 *$ & $0,36^{* *}$ & $19,76^{* *}$ & $431,86^{* *}$ & $1830427,96 *$ & $0,55^{* *}$ & $14,17 * *$ & $542,53 * *$ \\
\hline TEST & 4 & $527406,23 *$ & $0,30 \mathrm{~ns}$ & $23,83 * *$ & $205,76 \mathrm{~ns}$ & $433680,10 \mathrm{~ns}$ & $0,14 \mathrm{~ns}$ & $43,23 * *$ & $377,43 *$ & $1422367,45 \mathrm{~ns}$ & $0,27 \mathrm{~ns}$ & $15,60 * *$ & $383,14 *$ \\
\hline Hib vs Test & 1 & $5974944,25^{* *}$ & $10,74 * *$ & $976,63 * *$ & $719,72 * *$ & $4627080,08 * *$ & $6,34 * *$ & $985,69 * *$ & $684,13 *$ & $1688256,3 \mathrm{~ns}$ & $2,31 * *$ & $526,91 * *$ & $770,28^{*}$ \\
\hline Resíduo & 96 & 203491,48 & 0,28 & 2,57 & 87,84 & 477669,30 & 0,18 & 3,84 & 138,13 & 1040632,52 & 0,21 & 2,78 & 139,94 \\
\hline M. Geral & & 2488,66 & 2,56 & 68,02 & 237,56 & 4096,50 & 2,18 & 58,91 & 283,65 & 6213,98 & 2,39 & 66,85 & 272,66 \\
\hline M. Híbridos & & 2556,62 & 2,47 & 67,15 & 236,82 & 4156,31 & 2,11 & 58,04 & 282,92 & 6250,10 & 2,35 & 66,21 & 271,89 \\
\hline M. Test & & 1890,60 & 3,36 & 75,66 & 244,13 & 3570,20 & 2,80 & 66,60 & 290,05 & 5896,07 & 2,76 & 72,46 & 279,46 \\
\hline CV (\%) & & 18,12 & 20,72 & 2,35 & 3,94 & 16,87 & 19,65 & 3,32 & 4,14 & 16,41 & 19,15 & 2,49 & 4,33 \\
\hline
\end{tabular}

*, **, ns Significativo a 5\% e 1\% de probabilidade, e não significativo pelo teste de $\mathrm{F}$, respectivamente. 
(Híb. vs Test) para AP foi significativo para todas a localidades.

A variável floração masculina (FM) foi significativa $(\mathrm{P}<0,01)$ entre os híbridos e (Híb. vs Test) para todas as localidades, demonstrando assim a existência de genótipo (híbridos) que poderia ser liberado visando à precocidade.

Para a altura de planta (APl), observou-se significância para todas as localidades entres os híbridos $(\mathrm{P}<0,01)$. Para a fonte de variação (Híb. vs Test) observou-se significância para as localidades de Choré $(\mathrm{P}<0,05)$ e Yjhovy e para Capitán Miranda $(\mathrm{P}<0,01)$, mostrando maior diferença entre os genótipos e as testemunhas na última localidade mencionada.

Em relação à produtividade de grãos nos ensaios verifica-se que houve grande variação, com valores extremos de $2.488,66 \mathrm{~kg} \mathrm{ha}^{-1}$, no ensaio de Capitán Miranda, até $6.213,98 \mathrm{~kg} \mathrm{ha}^{-1}$, no ensaio de Yjhovy. Esta variação deve-se, principalmente, às características dos ambientes nos quais os híbridos foram avaliados principalmente ao fator de fertilidade do solo, tendo em vista que uma das localidades experimentais (Yjhovy) foi recentemente incluída na rede de experimentação constituindo-se, portanto, de um solo mais novo e fértil em termo de área agricultável. As médias de produtividade alcançadas pelos híbridos testados foram semelhantes às obtidas por Machado et al. (2002), para milho tipo amiláceo. Estes valores de produtividade foram muito superiores ao rendimento médio do cultivar Avati Morotî registrado no país (4.063 $\mathrm{kg} \mathrm{ha}^{-1}$ ) Machado (2013). Isto significa que os híbridos avaliados têm elevado potencial produtivo, e o desempenho deles depende grandemente dos ambientes em que foram avaliados.

Os dados da Tabela 3 apresentam os quadrados médios, médias e coeficientes de variação $(\mathrm{CV})$ para produtividade de grãos (PG), aspecto de plan- ta (AP), floração masculina (FM) e altura de planta (APl) avaliados em três ensaios de milho amiláceo; nela observam-se os valores da razão entre o maior e menor quadrado médio residual, em que se constata que este não foi superior a sete, obedecendo assim aos critérios de homogeneidade dos quadrados médios residuais para realização da análise de variância conjunta (Pimentel-Gomes, 1990).

Observam-se, na mesma Tabela 3, diferenças significativas ao $1 \%$ de nível de probabilidade por meio do teste $\mathrm{F}$, para todas as quatro características avaliadas, na fonte de variação genótipo (HIB), o que corrobora a existência de variabilidade detectada nas análises individuais. Para a fonte de variação (Híb. vs Test) as variáveis (PG) e (AP) foram significativos a $(\mathrm{P}<0,05)$, e para as variáveis de $(\mathrm{FM})$ e $(\mathrm{APl})$ foram significativos $(\mathrm{P}<0,01)$ confirmando a existência de genótipos precoce e de porte baixo que as testemunhas.

Para a fonte de variação ambiente, houve diferenças significativas, em nível de $1 \%$ de probabilidade, para todas as características avaliadas, o que indica variabilidade entre locais, fato importante para tornar o processo de indicação de genótipos superiores mais eficientes pressupondo que os locais envolvidos na experimentação sejam, de fato, representativos da região de cultivo e aptidão agrícola das cultivares ou dos híbridos de milho. Essas diferenças significativas encontradas entre os ambientes podem ser explicadas pelas diferenças de solo e climáticas entre os locais dos ensaios e por pequenas diferenças de tratos culturais que possam ter ocorrido desde o preparo do solo até a colheita, além de outras intempéries que possam ter ocorrido durante o ciclo da cultura em cada localidade onde foram instalados os experimentos.

Todas as características, com exceção da floração masculina, revelaram diferenças significativas, em nível de $5 \%$ de probabilidade pelo teste $\mathrm{F}$, para a 
Tabela 3. Quadrados médios, médias e coeficientes de variação $(\mathrm{CV})$ para produtividade de grãos em $\mathrm{kg} \mathrm{ha}^{-1}$ (PG), aspecto de planta (AP), floração masculina em dias (FM) e altura de planta em cm (APl) avaliados em três ensaios de milho amiláceo. Paraguai. Biênio 2011/12.

\begin{tabular}{|c|c|c|c|c|c|c|c|}
\hline \multirow[t]{2}{*}{ FV } & \multirow[t]{2}{*}{ GL } & \multicolumn{6}{|c|}{ QM } \\
\hline & & PG & AP & \multicolumn{2}{|c|}{ FM } & \multicolumn{2}{|l|}{ API } \\
\hline BLOCO/AMB & 6 & 1195083,40 & 0,58 & \multicolumn{2}{|c|}{1,77} & \multicolumn{2}{|l|}{1224,11} \\
\hline TRAT & 48 & $1567061,35 \quad * *$ & $1,06 * *$ & 106,08 & $* *$ & $835,52 *$ & $* *$ \\
\hline HIB & 43 & $1407320,46 \quad * *$ & $0,71 * *$ & 55,19 & $* *$ & $834,33 *$ & $* *$ \\
\hline TEST & 4 & 780354,41 & $0,56 *$ & 69,02 & $* *$ & 513,97 & ns \\
\hline HIB vs TEST & 1 & $11582747,44 *$ & $17,85^{*}$ & 2442,5 & $4 * *$ & 2172,85 & $* *$ \\
\hline AMBIENTES & 2 & $513197203,22 * *$ & $5,35 *$ & 3604,7 & $4 * *$ & 85172,62 & $2 * *$ \\
\hline TRAT $x$ AMB & 96 & 759618,98 & $0,29 *$ & 3,79 & ns & 171,43 & $*$ \\
\hline HIB x AMB & 86 & 765156,87 & $0,30 *$ & 3,05 & ns & 170,31 & $*$ \\
\hline TEST $\times$ AMB & 8 & $801549,69 \quad \mathrm{~ns}$ & $0,08 \mathrm{~ns}$ & 6,82 & $*$ & 226,18 & ns \\
\hline (HIB vs TEST) AMB & 2 & $353766,62 \quad \mathrm{~ns}$ & $0,77 *$ & 23,35 & $* *$ & 0,64 & ns \\
\hline RESIDUO & 288 & 573931,10 & 0,22 & 3,0 & & 121,97 & \\
\hline Média & & 4266,38 & 2,38 & 64,5 & & 264,63 & \\
\hline Médias Híbridos & & 4321,01 & 2,31 & 63,8 & & 263,88 & \\
\hline Médias Testemunhas & & 3785,62 & 2,97 & 71,5 & & 271,21 & \\
\hline CV (\%) & & 17,75 & 19,95 & 2,7 & & 4,17 & \\
\hline Razão (>QMr/<QMr) & & 5,11 & 1,53 & 1,4 & & 1,59 & \\
\hline
\end{tabular}

**, * , ns Significativo a $1 \%$, a $5 \%$ de probabilidade e não significativo pelo teste de $\mathrm{F}$, respectivamente.

fonte de variação interação HIB x AMB. Resultados semelhantes foram obtidos por Souza et al. (2002), e Cardoso et al. (2003). A significância verificada na fonte de variação interação HIB x AMB mostra que houve diferença no comportamento dos genótipos, na manifestação ambiental, e que, de um local para outro, os genótipos mostraram manifestação fenotípica inconsistente perante as variações ambientais.

A significância da interação HIB x AMB adverte sobre a necessidade de se identificarem as cultivares com adaptação mais específica, ou que sejam menos afetadas pela variação dos ambientes. A interação ocorre quando há respostas diferenciadas dos genótipos em relação ao ambiente (Cruz et al., 2012). Segundo Borém e Miranda (2013), a interação Ge- nótipos x Ambientes, de maneira geral, constitui um importante fator e um desafio para melhoristas e agrônomos que atuam na recomendação de cultivares.

Para produção de grão (PG) foi formado um grupo na localidade de Choré e Capitán Miranda e dois grupos em Yjhovy. Os híbridos com média mais alta em Yjhovy foram 33, 17, 24 e 26; na localidade de Capitán Miranda, 31, 15, 28 e 35; e em Choré, 32, 31, 19 e 27 (Tabela 3). Também pode-se observar que as médias das testemunhas foram amplamente superadas pelas médias dos melhores híbridos para todas as localidades.

Pode-se observar que somente o híbrido 31 foi superior nas localidades Capitán Miranda e Choré. Os piores híbridos para produção de grãos foram os ge- 
Tabela 4. Agrupamento de Scott-Knott para produtividade de grãos em $\mathrm{kg} \mathrm{ha}^{-1}(\mathrm{PG})$, aspecto de planta (AP), floração masculina em dias (FM) e altura de planta em cm (APl) avaliados em três ensaios de milho amiláceo. Paraguai. Biênio 2011/12.

\begin{tabular}{|c|c|c|c|c|c|c|c|c|c|c|c|c|c|c|c|c|c|c|c|c|c|c|c|c|}
\hline \multirow{2}{*}{ GEN } & \multicolumn{8}{|c|}{ CAPITAN MIRANDA } & \multicolumn{8}{|c|}{ CHORÉ } & \multicolumn{8}{|c|}{ YJHOVY } \\
\hline & PG & & AP & & $\mathrm{FM}$ & & APl & & PG & & AP & & FM & & APl & & $\mathrm{P}$ & & AP & & $\mathrm{M}$ & & APl & \\
\hline 1 & 45,67 & $\mathrm{a}$ & 2,67 & $\mathrm{a}$ & 69,33 & $\mathrm{~b}$ & 37,13 & $\mathrm{a}$ & 02,00 & $\mathrm{a}$ & 2,83 & $\mathrm{a}$ & 58,67 & $\mathrm{~b}$ & 67,10 & b & 47,50 & $\mathrm{~b}$ & 2,50 & $\mathrm{a}$ & 66,67 & $\mathrm{a}$ & 259,33 & \\
\hline 2 & 2635,67 & $\mathrm{a}$ & 2,67 & $\mathrm{a}$ & 66,00 & $\mathrm{c}$ & 247,77 & a & & a & 2,50 & $\mathrm{a}$ & 58,67 & $\mathrm{~b}$ & & & 6912,60 & $\mathrm{a}$ & 2,83 & $\mathrm{a}$ & 65,00 & b & 266,43 & \\
\hline 3 & 2467,67 & $\mathrm{a}$ & 2,83 & $\mathrm{a}$ & 65,67 & 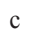 & 36,33 & $\mathrm{~b}$ & 3764,00 & $\mathrm{a}$ & 2,33 & $\mathrm{a}$ & 58,00 & $\mathrm{~b}$ & 7,77 & & 5399,70 & $\mathrm{~b}$ & 3,00 & $\mathrm{a}$ & 66,33 & $\mathrm{a}$ & 1,33 & \\
\hline 4 & 35,33 & $\mathrm{a}$ & 2,50 & $\mathrm{a}$ & 68,33 & t & 229,03 & $\mathrm{~b}$ & 398,00 & $\mathrm{a}$ & 2,00 & $\mathrm{~b}$ & 57,67 & $\mathrm{~b}$ & 270,33 & & 6405,80 & $\mathrm{a}$ & 2,00 & $\mathrm{~b}$ & 6,00 & $\mathrm{~b}$ & 41,77 & \\
\hline 5 & 03,33 & $\mathrm{a}$ & 3,33 & $\mathrm{a}$ & 68,67 & $\mathrm{~b}$ & 27,47 & $\mathrm{~b}$ & 07,33 & 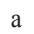 & 2,83 & $\mathrm{a}$ & 60,67 & $\mathrm{a}$ & 64,23 & & 334,77 & b & 3,33 & $\mathrm{a}$ & 8,33 & $\mathrm{a}$ & 8,10 & \\
\hline 6 & 2629,00 & $\mathrm{a}$ & 2,67 & $\mathrm{a}$ & 71,33 & $\mathrm{a}$ & 232,67 & $\mathrm{~b}$ & 230,33 & $\mathrm{a}$ & 2,50 & $\mathrm{a}$ & 60,67 & a & 270,33 & b & 5859,37 & $\mathrm{~b}$ & 2,67 & $\mathrm{a}$ & 68,67 & $\mathrm{a}$ & 2,30 & \\
\hline 7 & 2500,33 & $\mathrm{a}$ & 2,50 & $\mathrm{a}$ & 69,67 & $\mathrm{~b}$ & 233,53 & $\mathrm{~b}$ & 4121,67 & $\mathrm{a}$ & 2,33 & $\mathrm{a}$ & 60,67 & $\mathrm{a}$ & & & & $\mathrm{b}$ & 2,83 & $\mathrm{a}$ & 65,67 & $\mathrm{~b}$ & 1,77 & \\
\hline 8 & 2241,00 & $\mathrm{a}$ & 3,17 & $\mathrm{a}$ & 67,67 & 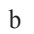 & 236,00 & $\mathrm{~b}$ & 4094,67 & a & 2,00 & $\mathrm{~b}$ & 57,67 & $\mathrm{~b}$ & & & & b & 2,50 & $\mathrm{a}$ & 66,67 & $\mathrm{a}$ & 76,20 & \\
\hline 9 & 1,00 & $\mathrm{a}$ & 2,33 & $\mathrm{~b}$ & 63,00 & 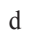 & $0 /$ & 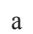 & n & a & 2,33 & $\mathrm{a}$ & 55,33 & 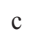 & & & 13 & & 2,50 & $\mathrm{a}$ & 3,33 & $\mathrm{~b}$ & 77 & \\
\hline 10 & 21,33 & $\mathrm{a}$ & 2,33 & b & 63,67 & 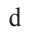 & 3,90 & b & 3183,00 & $\mathrm{a}$ & 2,33 & $\mathrm{a}$ & 54,33 & 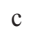 & 5,23 & & 632,03 & b & 2,50 & $\mathrm{a}$ & 3,33 & $\mathrm{~b}$ & 43 & \\
\hline 11 & 64,67 & $\mathrm{a}$ & 2,33 & $\mathrm{~b}$ & 70,00 & $\mathrm{~b}$ & 234,03 & $\mathrm{~b}$ & 4624,67 & $\mathrm{a}$ & 2,17 & $\mathrm{a}$ & 61,00 & a & 8,33 & a & 576,87 & $\mathrm{a}$ & 1,50 & $\mathrm{~b}$ & 68,00 & 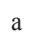 & 2,33 & \\
\hline 12 & 22,67 & $\mathrm{a}$ & 2,67 & $\mathrm{a}$ & 68,33 & $\mathrm{~b}$ & & 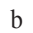 & & a & 1,67 & $\mathrm{~b}$ & 59,33 & $\mathrm{~b}$ & & & & & 2,00 & $\mathrm{~b}$ & 67,33 & a & & \\
\hline 13 & 57,33 & $\mathrm{a}$ & 2,50 & $\mathrm{a}$ & 67,33 & $\mathrm{~b}$ & 23047 & $\mathrm{~b}$ & 305267 & $a$ & 2,00 & $\mathrm{~b}$ & 59,00 & $\mathrm{~b}$ & & & & p & 2,17 & $\mathrm{~b}$ & 65,67 & $\mathrm{~b}$ & & $\mathrm{c}$ \\
\hline 14 & 33 & $\mathrm{a}$ & 2,33 & $\mathrm{~b}$ & 63,33 & 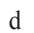 & & 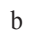 & & a & 2,17 & $\mathrm{a}$ & 55,00 & 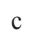 & & & & & 2,33 & $\mathrm{a}$ & 3,00 & $\mathrm{~b}$ & & \\
\hline 15 & 5,33 & $\mathrm{a}$ & 2,00 & b & 62,00 & d & & 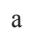 & 92,33 & a & 2,50 & $\mathrm{a}$ & 54,33 & & & & 87 & & 2,33 & a & 4,00 & b & ,97 & \\
\hline 16 & & $\mathrm{a}$ & 2,83 & $\mathrm{a}$ & 72,00 & 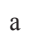 & & a & & $\mathrm{a}$ & 2,00 & $\mathrm{~b}$ & 60,67 & a & & & & & 2,00 & $\mathrm{~b}$ & 69,33 & a & & \\
\hline 17 & 900 & $\mathrm{a}$ & 2,67 & $\mathrm{a}$ & 71,00 & 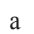 & & $\mathrm{a}$ & & $\mathrm{a}$ & 1,67 & $\mathrm{~b}$ & 60,33 & 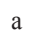 & & & & & 2,50 & $\mathrm{a}$ & 69,67 & $\mathrm{a}$ & & $\mathrm{a}$ \\
\hline 18 & 38,00 & $\mathrm{a}$ & 3,00 & $\mathrm{a}$ & 70,00 & b & & $\mathrm{a}$ & 4185,00 & $\mathrm{a}$ & 2,00 & $\mathrm{~b}$ & 59,33 & $\mathrm{~b}$ & & & & b & 3,00 & $\mathrm{a}$ & 68,00 & $\mathrm{a}$ & & $\mathrm{a}$ \\
\hline 19 & & $\mathrm{a}$ & 2,17 & $\mathrm{~b}$ & 69,33 & $\mathrm{~b}$ & & a & & a & 1,83 & $\mathrm{~b}$ & 59,33 & $\mathrm{~b}$ & & & & & 2,17 & $\mathrm{~b}$ & 7,33 & $\mathrm{a}$ & & b \\
\hline 20 & 5,33 & . & 2,83 & $\mathrm{a}$ & 71,33 & $\mathrm{a}$ & & & & & 2,50 & $\mathrm{a}$ & 62,00 & a & & & & & 3,00 & $\mathrm{a}$ & 8,33 & & & \\
\hline 21 & 7,00 & $\mathrm{a}$ & 2,33 & $\mathrm{~b}$ & 72,33 & " & & a & & ( & 2,00 & $\mathrm{~b}$ & 63,00 & & & & & & 2,67 & 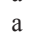 & 70,00 & & & $\mathrm{a}$ \\
\hline 22 & 16,33 & $\mathrm{a}$ & 2,67 & $\mathrm{a}$ & 72,67 & 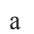 & 2 & b & & $\mathrm{a}$ & 2,50 & $\mathrm{a}$ & 63,67 & 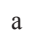 & & & & 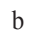 & 2,17 & $\mathrm{~b}$ & 71,00 & $\mathrm{a}$ & & b \\
\hline 23 & 2683,33 & $\mathrm{a}$ & 2,00 & $\mathrm{~b}$ & 69,00 & b & 20057 & 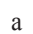 & 33 & a & 1,67 & $\mathrm{~b}$ & 58,33 & b & 10 & & 60 & 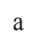 & 1,83 & $\mathrm{~b}$ & 67,33 & $\mathrm{a}$ &, 67 & $\mathrm{c}$ \\
\hline 24 & & $\mathrm{a}$ & 2,67 & $\mathrm{a}$ & 69,33 & $\mathrm{~b}$ & & $\ldots$ & & a & 2,00 & $\mathrm{~b}$ & 58,00 & $\mathrm{~b}$ & & & & a & 2,50 & $\mathrm{a}$ & 8,00 & $\mathrm{a}$ & & b \\
\hline 25 & & 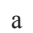 & 2,83 & $\mathrm{a}$ & 68,00 & $\mathrm{~b}$ & & b & & & 3 & $\mathrm{~b}$ & 57,33 & b & & & & & 2,00 & $\mathrm{~b}$ & 5,00 & $\mathrm{U}$ & & \\
\hline 26 & 67 & $\mathrm{a}$ & 1,33 & $\mathrm{~b}$ & 64,67 & 列 & & 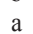 & & & 1,83 & $\mathrm{~b}$ & ( & 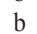 & & & & & 2,33 & $\mathrm{a}$ & 5,33 & $\mathrm{~b}$ & & \\
\hline 27 & 2,00 & $\mathrm{a}$ & 2,50 & $\mathrm{a}$ & 66,33 & . & & 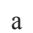 & & $\mathrm{a}$ & 1,50 & $\mathrm{~b}$ & 55,33 & 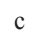 & & & & & 1,83 & $\mathrm{~b}$ & 65,67 & $\mathrm{~b}$ & & b \\
\hline 28 & 01,00 & $\mathrm{a}$ & 2,33 & b & 66,00 & 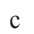 & & 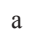 & & 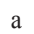 & 2,50 & $\mathrm{a}$ & 57,33 & $\mathrm{~b}$ & & & & b & 2,33 & $\mathrm{a}$ & 64,00 & o &, 77 & b \\
\hline 29 & & 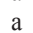 & 2,67 & $\mathrm{a}$ & 62,67 & $d$ & & b & & 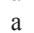 & 2,00 & $\mathrm{~b}$ & & $\mathrm{c}$ & & & & $\mathrm{b}$ & 2,50 & $\mathrm{a}$ & 33 & $\mathrm{~b}$ & & $\mathrm{c}$ \\
\hline 30 & 3 & $\mathrm{a}$ & 2,50 & $\mathrm{a}$ & 62,67 & d & & $\mathrm{a}$ & & a & 1,83 & $\mathrm{~b}$ & & $\mathrm{c}$ & & & & & 2,83 & $\mathrm{a}$ & 00 & b & & \\
\hline 31 & 7,00 & 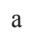 & 2,00 & b & 69,00 & 0 & & & & & 2,33 & a & 59 & 0 & & & & & 2,33 & $\mathrm{a}$ &, 33 & & & \\
\hline 3 & 0,00 & $\mathrm{a}$ & 2,00 & $\mathrm{~b}$ & 65,67 & 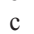 & & a & & a & 1,50 & $\mathrm{~b}$ & 56,67 & $\mathrm{c}$ & & & & & 1,83 & $\mathrm{~b}$ & 64,00 & $\mathrm{~b}$ & & $\mathrm{c}$ \\
\hline 33 & 2,33 & $\mathrm{a}$ & 1,67 & $\mathrm{~b}$ & 66,33 & 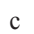 & & $\mathrm{a}$ & & $\mathrm{a}$ & 2,50 & 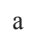 & 55,67 & c & & & & & 2,67 & $\mathrm{a}$ & 68,67 & $\mathrm{a}$ & & b \\
\hline 34 & & $\mathrm{a}$ & 2,67 & $\mathrm{a}$ & 64,67 & - & & - & & 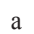 & 2,17 & s & & $\mathrm{c}$ & & & & a & 2,00 & $\mathrm{~b}$ & 64,00 & $\mathrm{~b}$ & & $\mathrm{c}$ \\
\hline 2 & & $\mathrm{a}$ & 2,00 & $\mathrm{~b}$ & 64,33 & - & & - & & 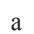 & 2,17 & 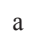 & & $\mathrm{b}$ & & & & & 3,00 & $\mathrm{a}$ & 64,33 & - & & \\
\hline 2 & & 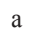 & 2,5 & $\mathrm{a}$ & 61,67 & d & & b & & $\mathrm{a}$ & 2,33 & $\mathrm{a}$ & 54,00 & $\mathrm{c}$ & & & & $\mathrm{a}$ & 2,33 & $\mathrm{a}$ & 63,00 & b & 10 & \\
\hline 2 & 67 & a & 2,50 & $\mathrm{a}$ & 67,3 & $\mathrm{~b}$ & & b & & 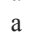 & & 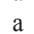 & 58,00 & b & & & & a & 2,00 & $\mathrm{~b}$ & 65,67 & $\mathrm{~b}$ & & \\
\hline 3 & 6,33 & $\mathrm{a}$ & 2,33 & $\mathrm{~b}$ & 64,67 & $c$ & & 0 & & $\mathrm{a}$ & & a & 56,00 & c & & & & $\mathrm{b}$ & 2,50 & $\mathrm{a}$ & 65,33 & D & & c \\
\hline 39 & 2195,33 & $\mathrm{a}$ & 2,50 & $\mathrm{a}$ & 66,00 & $\mathrm{c}$ & & $\mathrm{a}$ & & $\mathrm{a}$ & 2,33 & $\mathrm{a}$ & 57,33 & $\mathrm{~b}$ & & & & $\mathrm{a}$ & 2,50 & $\mathrm{a}$ & 64,67 & $\mathrm{~b}$ & 80 & b \\
\hline 40 & 7 & a & 2,17 & $\mathrm{~b}$ & 61,67 & 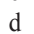 & & 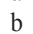 & & 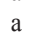 & 2,00 & $\mathrm{~b}$ & 51,67 & $\mathrm{c}$ & & & & 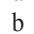 & 2,67 & $\mathrm{a}$ & 63,33 & $\mathrm{~b}$ & & $\mathrm{c}$ \\
\hline 41 & & $\mathrm{a}$ & 2,3 & $\mathrm{~b}$ & 68,33 & t & & a & & $\mathrm{a}$ & 1,33 & $\mathrm{~b}$ & & a & & & & a & 1,67 & $\mathrm{~b}$ & 66,67 & $\mathrm{a}$ & & \\
\hline in & & $\mathrm{a}$ & 年 & 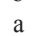 & (1) & 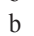 & & b & & $\mathrm{a}$ & 1,83 & $\mathrm{~b}$ & & b & & & & & 2,17 & $\mathrm{~b}$ & 68,33 & & & \\
\hline 4 & & $\mathrm{a}$ & 2,50 & $\mathrm{a}$ & 67,33 & $\mathrm{~b}$ & & 0 & & $\mathrm{a}$ & 1,67 & $\mathrm{~b}$ & & b & & & & a & 1,50 & $\mathrm{~b}$ & 67,67 & $\mathrm{a}$ & & b \\
\hline 44 & 2327,00 & $\mathrm{a}$ & 2,67 & $\mathrm{a}$ & 67,00 & $\mathrm{~b}$ & & $\mathrm{~b}$ & & $\mathrm{a}$ & 2,00 & $\mathrm{~b}$ & 59,67 & $\mathrm{~b}$ & & $\mathrm{~b}$ & & $\mathrm{a}$ & 1,67 & $\mathrm{~b}$ & 67,00 & $\mathrm{a}$ & 6,23 & c \\
\hline 45 & 2194,33 & $\mathrm{a}$ & 3,50 & $\mathrm{a}$ & 77,00 & 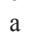 & & a & & 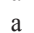 & 2,83 & . & 70,33 & $\mathrm{a}$ & & & & a & 2,83 & a & 74,66 & $\mathrm{a}$ & & \\
\hline 46 &, 33 & $\mathrm{~b}$ & 3,16 & $\mathrm{~b}$ & 73,33 & $\mathrm{~b}$ & & 0 & & 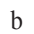 & 3,00 & $\mathrm{~b}$ & 60,66 & b & & D & & b & 2,83 & $\mathrm{~b}$ & 69,33 & D & 9,23 & \\
\hline 2. & & $\mathrm{c}$ & 3,00 & $\mathrm{c}$ & & 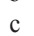 & & $\mathrm{c}$ & & ( & 2,66 & & & . & & & & $c$ & 2,33 & $\mathrm{c}$ & 71,33 & c & & \\
\hline 48 & 00 & d & 3,83 & d & 80,00 & a & & d & & d & 3,00 & d & & d & & & & d & 3,16 & d & 74,66 & d & & d \\
\hline 49 & 1884,66 & $\mathrm{e}$ & 3,33 & $\mathrm{e}$ & 73,66 & 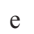 & 241,56 & $\mathrm{e}$ & 3030,33 & $\mathrm{e}$ & 2,50 & & 65,33 & $\mathrm{e}$ & 295,00 & 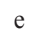 & 5461,03 & 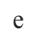 & 2,66 & $\mathrm{e}$ & 72,33 & 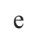 & 278,53 & \\
\hline
\end{tabular}

nótipos 5, 10 e 22 para a localidade de Yjhovy. Como nas demais localidades não houve diferença significativa, as médias obtidas para os mesmos genótipos também foram as mais baixas.
Para variável aspecto de planta (AP), os híbridos 26, 33, 35 e 15; 41, 19, 27 e 32; 11, 43, 41 e 32 foram os que apresentaram menores valores para as localidades de Capitán Miranda, Choré e Yjhovy, 
respectivamente. Estes foram os genótipos que apresentaram melhores aspectos de planta. É válido ressaltar que nem sempre aquelas plantas de melhor aspecto foram as que apresentaram maior produtividade, uma vez que muitos fatores são considerados para o aspecto de planta. O híbrido 5 foi o que apresentou maior valor em todos os locais sendo o híbrido de menor produção de grão. É importante destacar que as maiorias das testemunhas apresentaram valores de AP alta, com exceção do genótipo 47 , que apresentou o menor valor, isso porque o material é mais promissor.

$\mathrm{Na}$ variável floração masculina (FM), os híbridos 40, 36, 30 e 29 foram os que apresentaram menos dias para florescimento em todos os locais. $\mathrm{O}$ genótipo 40 foi o de maior precocidade entre todos. Considerando a variável dias para o florescimento, esta apresentou valores médios de 51,67, 61,67 e 63,33 para as localidades de Choré, Capitán Miranda e Yjhovy, respectivamente, podendo esta variação estar relacionado à localização geográfica entre as localidades. Isso retrata o fato de as condições entre os locais serem bem contrastantes entre si. É possível ver também que as testemunhas foram mais tardias que os híbridos mencionados, atingido assim o objetivo do programa que é obter cultivares mais precoces.

O comportamento dos genótipos para altura de planta (APl) foram diferentes para todos os ambientes avaliados, sendo os híbridos 44, 4, 43 e 12; 15, 5, 1 e 44; 4, 14, 13 e 1 para as localidades de Capitán Miranda, Choré e Yjhovy, respectivamente, o que apresentaram menores valores de altura. As maiores alturas de planta foram apresentadas pelos híbridos 21 e 18 para todas as localidades. As testemunhas também foram os que apresentaram as maiores altura de planta.

A existência de respostas diferenciadas de genótipos às variações ambientais tem sido frequentemente constatada. Essa interação, além de influenciar os ganhos genéticos, dificulta a recomendação para mais de um ambiente. Neste sentido, realizouse procedimento para obtenção das estimativas da parte complexa das interações (\% C), em diferentes genótipos de milho amiláceo avaliados em três ambientes. Foi constatada interação significativa na fonte de variação GxA para as características PG, AP e APl (Tabela 4). Apenas a característica FM não expressou diferenças significantes para esta fonte de variação.

Tabela 5. Estimativas das Interações Complexas $(\% \mathrm{C})$, para produtividade de grãos $(\mathrm{PG})$, aspecto de planta (AP), floração masculina (FM) e altura de planta (APl) avaliados em três ensaios de milho amiláceo. Paraguai. Biênio 2011/12.

\begin{tabular}{lcc}
\hline \multicolumn{1}{c}{ Características } & $\begin{array}{c}\text { Estimativas Percentuais } \\
\text { das Interações Complexas }\end{array}$ & $\begin{array}{c}\text { Estimativas Percentuais } \\
\text { das Interações Complexas }\end{array}$ \\
\hline $\mathrm{PG}^{*}$ & 62,01 & 55,27 \\
$\mathrm{AP}^{*}$ & 98,61 & 87,95 \\
$\mathrm{FM}^{\mathrm{NS}}$ & 63,09 & 19,28 \\
$\mathrm{APl}^{*}$ & 77,50 & 51,13 \\
\hline
\end{tabular}

1. Método de Robertson (1959); ${ }^{2}$. Método de Cruz e Castoldi (1991).

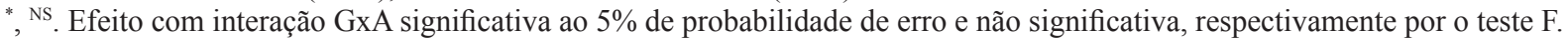


Avaliando-se os dados da Tabela 5, notase que a maioria das características revelou interação complexa superior a 50\%. Apenas a característica floração masculina apresentou valor inferior a $50 \%$ pelo método de Cruz e Castoldi (1991), o que é bastante coerente tendo em vista que, de fato, não houve manifestação da interação GxA para esta característica. Esses resultados foram similares aos obtidos por Garbuglio et al. (2007) e Ribeiro e Almeida (2011). A interação de tipo complexa indica a inconsistência da superioridade de genótipos com a variação ambiental, ou seja, haverá genótipos com desempenho superior em um ambiente, mas não em outro, tornando mais difícil a seleção e/ou a recomendação destes (Cruz et al., 2004 citado por Candido et al., 2011).

Os resultados obtidos comprovam que a maior parte da interação foi do tipo complexa. Desse modo, as diferenças entre os ambientes Capitán Miranda, Choré e Yjhovy foram suficientemente elevadas para o estabelecimento de programas de melhoramento próprios para cada ambiente, considerando os caracteres estudados.

Segundo Carvalho et al. (2002), a seleção e a recomendação de genótipos mais produtivos são objetivos básicos dos programas de melhoramento genético de qualquer espécie cultivada. $\mathrm{O}$ processo de seleção é frequentemente realizado pelo desempenho dos genótipos em diferentes ambientes. Contudo, a decisão de recomendação de novas cultivares normalmente é dificultada pela ocorrência da interação genótipos x ambientes.

Tendo em vista a ocorrência de interação híbridos x ambientes significativos torna-se necessário realizar estudos pormenorizados e detalhados sobre o desempenho dos genótipos nos diferentes ambientes. Pelas estimativas da estatística Pi, de Lin e Binns (1988), expostas na Tabela 6, verificase que os híbridos 41 e 24, respectivamente, foram os que contiveram os menores valores de Pi geral e maior média para produtividade de grão (PG), sendo, desta forma, considerados os mais adaptados e estáveis.

A alta correlação entre média e a medida da estabilidade fenotípica é uma característica do método de Lin e Binns (1988), pois associa estabilidade com a capacidade dos genótipos de apresentar o menor desvio em relação ao máximo, em todos os ambientes do estudo. Essa é considerada a maior vantagem desse método, pois consegue identificar os genótipos mais estáveis, sempre entre os mais produtivos, como observado também por Cargnelutti Filho et al. (2007), para produtividade na cultura de milho.

Para a variável aspecto de planta (AP), considerando o valor do Pi, os híbridos 41 e 32 apresentaram os melhores comportamentos, já que as notas de AP consideraram valores crescentes, sendo a menor média melhor, e o híbrido 41 apresentou menor média e maior Pi, concordando assim com o mais estável e de melhor aspecto da planta.

Considerando a floração masculina e altura de planta, os genótipos mais precoces não foram os mais produtivos e nem de menor porte. Visando o objetivo do programa de obter híbridos estáveis, precoces e de porte médio pode-se dizer que, selecionando o híbrido mais estável (41), pode-se atingir o mencionado acima, já que a média de produção do híbrido 41 também foi a maior dentre as últimas cultivares disponibilizadas pelo IPTA, para este tipo de grão (Machado, 2013). 
Tabela 6. Estimativas dos parâmetros de estabilidade propostas por Lin e Binns (1988) para produtividade de grãos em kg ha-1(PG), aspecto de planta (AP), floração masculina em dias (FM) e altura de planta em cm (APl) avaliados em três ensaios de milho amiláceo. Paraguai. Biênio 2011/12.

\begin{tabular}{|c|c|c|c|c|c|c|c|c|c|c|c|}
\hline \multirow{2}{*}{ GEN } & \multicolumn{2}{|c|}{ PG } & \multirow{2}{*}{ GEN } & \multicolumn{2}{|c|}{ AP } & \multirow{2}{*}{ GEN } & \multicolumn{2}{|c|}{ FM } & \multirow{2}{*}{ GEN } & \multicolumn{2}{|c|}{ AP1 } \\
\hline & Média & $\mathrm{Pi}$ & & Média & $\mathrm{Pi}$ & & Média & $\mathrm{Pi}$ & & Média & $\mathrm{Pi}$ \\
\hline 41 & 4926,78 & 146872,90 & 5 & 3,17 & 0,00 & 22 & 69,11 & 0,00 & 21 & 288,31 & 0,62 \\
\hline 24 & 4840,90 & 204434,48 & 20 & 2,78 & 0,08 & 21 & 68,44 & 0,26 & 18 & 286,77 & 7,49 \\
\hline 17 & 4776,99 & 256345,46 & 3 & 2,72 & 0,10 & 20 & 67,22 & 1,94 & 17 & 280,19 & 43,39 \\
\hline 33 & 4916,46 & 320325,04 & 2 & 2,67 & 0,13 & 16 & 67,33 & 2,04 & 19 & 281,38 & 58,15 \\
\hline 19 & 4709,90 & 335128,06 & 18 & 2,67 & 0,15 & 17 & 67,00 & 2,61 & 20 & 279,27 & 60,90 \\
\hline 27 & 4690,21 & 339476,01 & 6 & 2,61 & 0,17 & 6 & 66,89 & 2,70 & 16 & 273,73 & 121,58 \\
\hline 32 & 4881,74 & 353324,47 & 1 & 2,67 & 0,19 & 11 & 66,33 & 3,87 & 31 & 273,43 & 141,71 \\
\hline 43 & 4633,76 & 386537,08 & 7 & 2,56 & 0,20 & 5 & 65,89 & 5,35 & 41 & 272,26 & 153,36 \\
\hline 11 & 4622,07 & 392235,38 & 8 & 2,56 & 0,24 & 18 & 65,78 & 5,81 & 33 & 269,88 & 200,28 \\
\hline 4 & 4646,38 & 421444,65 & 39 & 2,44 & 0,27 & 19 & 65,33 & 7,22 & 39 & 269,17 & 206,35 \\
\hline 23 & 4597,09 & 435487,64 & 24 & 2,39 & 0,31 & 31 & 65,22 & 7,61 & 27 & 269,00 & 206,72 \\
\hline 26 & 4726,36 & 465693,77 & 29 & 2,39 & 0,31 & 7 & 65,33 & 7,74 & 8 & 269,00 & 225,89 \\
\hline 44 & 4524,02 & 500756,98 & 22 & 2,44 & 0,32 & 41 & 65,11 & 8,11 & 7 & 267,84 & 230,21 \\
\hline 34 & 4499,78 & 500849,10 & 9 & 2,39 & 0,32 & 12 & 65,00 & 8,50 & 28 & 268,48 & 231,06 \\
\hline 16 & 4460,34 & 517889,94 & 10 & 2,39 & 0,32 & 24 & 65,11 & 8,70 & 2 & 269,03 & 285,75 \\
\hline 42 & 4442,63 & 524586,55 & 30 & 2,39 & 0,32 & 42 & 65,11 & 8,78 & 30 & 268,32 & 299,74 \\
\hline 15 & 4592,84 & 539947,88 & 36 & 2,39 & 0,32 & 1 & 64,89 & 9,15 & 32 & 266,30 & 301,92 \\
\hline 25 & 4402,89 & 583515,63 & 38 & 2,39 & 0,32 & 23 & 64,89 & 9,22 & 24 & 265,80 & 308,75 \\
\hline 20 & 4403,46 & 612737,28 & 28 & 2,39 & 0,35 & 43 & 64,78 & 9,72 & 11 & 264,90 & 311,95 \\
\hline 31 & 4767,93 & 619440,17 & 21 & 2,33 & 0,36 & 44 & 64,56 & 10,69 & 9 & 264,99 & 352,31 \\
\hline 30 & 4356,41 & 729004,75 & 35 & 2,39 & 0,39 & 13 & 64,00 & 13,11 & 35 & 262,59 & 384,58 \\
\hline 2 & 4362,09 & 731051,71 & 14 & 2,28 & 0,41 & 8 & 64,00 & 13,30 & 22 & 261,18 & 393,85 \\
\hline 36 & 4219,82 & 804317,15 & 40 & 2,28 & 0,42 & 4 & 64,00 & 13,30 & 38 & 261,23 & 432,25 \\
\hline 39 & 4209,78 & 809894,71 & 17 & 2,28 & 0,42 & 25 & 63,78 & 14,48 & 3 & 261,81 & 437,26 \\
\hline 38 & 4304,81 & 812065,03 & 42 & 2,28 & 0,44 & 37 & 63,67 & 14,83 & 43 & 258,92 & 459,73 \\
\hline 12 & 4236,01 & 824598,56 & 34 & 2,28 & 0,44 & 3 & 63,33 & 17,15 & 26 & 262,38 & 464,84 \\
\hline 6 & 4239,57 & 895243,26 & 16 & 2,28 & 0,45 & 2 & 63,22 & 17,57 & 34 & 258,66 & 467,95 \\
\hline 37 & 4160,03 & 900547,57 & 37 & 2,28 & 0,45 & 33 & 63,56 & 18,26 & 6 & 258,43 & 488,73 \\
\hline 28 & 4319,88 & 904521,69 & 13 & 2,22 & 0,46 & 26 & 62,78 & 20,76 & 40 & 258,26 & 506,47 \\
\hline 9 & 4225,71 & 942075,55 & 15 & 2,28 & 0,48 & 39 & 62,67 & 20,78 & 37 & 256,92 & 534,13 \\
\hline 21 & 4153,02 & 949944,75 & 25 & 2,22 & 0,50 & 28 & 62,44 & 22,26 & 12 & 257,32 & 545,12 \\
\hline 40 & 4275,03 & 970672,57 & 31 & 2,22 & 0,50 & 27 & 62,44 & 23,00 & 23 & 261,11 & 561,56 \\
\hline 14 & 4143,29 & 1004903,91 & 4 & 2,17 & 0,53 & 35 & 62,44 & 23,15 & 15 & 257,36 & 562,91 \\
\hline 1 & 4031,72 & 1101075,24 & 33 & 2,28 & 0,56 & 32 & 62,11 & 24,50 & 29 & 255,82 & 565,53 \\
\hline 35 & 4218,88 & 1159025,60 & 12 & 2,11 & 0,60 & 38 & 62,00 & 25,81 & 42 & 256,96 & 574,21 \\
\hline 13 & 4022,74 & 1188031,98 & 19 & 2,06 & 0,62 & 34 & 61,67 & 27,80 & 36 & 255,67 & 580,83 \\
\hline 7 & 4002,18 & 1311628,38 & 44 & 2,11 & 0,65 & 9 & 60,56 & 36,94 & 13 & 255,02 & 619,96 \\
\hline 3 & 3877,12 & 1472891,74 & 27 & 1,94 & 0,79 & 14 & 60,44 & 37,70 & 25 & 254,24 & 653,35 \\
\hline 18 & 3933,31 & 1477783,06 & 11 & 2,00 & 0,80 & 10 & 60,44 & 37,81 & 5 & 253,27 & 662,77 \\
\hline 22 & 3778,20 & 1559256,17 & 23 & 1,83 & 0,90 & 30 & 60,33 & 38,91 & 1 & 254,52 & 665,14 \\
\hline 29 & 3827,09 & 1607675,73 & 43 & 1,89 & 0,90 & 15 & 60,11 & 41,65 & 44 & 252,44 & 677,19 \\
\hline 8 & 3571,53 & 2378024,18 & 32 & 1,78 & 0,97 & 29 & 59,56 & 45,85 & 10 & 251,86 & 757,87 \\
\hline 10 & 3412,12 & 2555907,43 & 26 & 1,83 & 1,00 & 36 & 59,56 & 46,41 & 14 & 249,78 & 836,10 \\
\hline 5 & 3181,81 & 2833109,10 & 41 & 1,78 & 1,00 & 40 & 58,89 & 53,96 & 4 & 247,04 & 1001,90 \\
\hline
\end{tabular}




\section{Conclusões}

1- A significância na fonte de variação GxA para a maioria das características avaliadas confirma a existência de interação genótipos por ambientes;

2- A maioria das características (PG, AP, APl) revelou a maior parte da interação do tipo complexa. Apenas a floração masculina (FM) apresentou interação do tipo simples; e

3- Os genótipos mais adaptados e estáveis pelo método de Lin e Binns foram os genótipos 41 e 24.

\section{Agradecimentos}

Ao IPTA (Instituto Paraguayo de Tecnología Agraria), INBIO (Instituto de Biotecnología Agrícola), pela ajuda financeira.

Ao Engenheiro Orlando Noldín e a todos os técnicos do Programa de Investigación Maíz, Sorgo y Girasol, Juan Morel (Juanito), Rodolfo Schopfer, Mario Díaz, Victorio Báez y Rubén Viera.

A todos os colegas do laboratório de bioinformática, Angélica, Rafael, Ricardo, Vinicius, Haroldo, Isabela, Gabi, Gislâyne e Marciane, que de algumas e outras maneiras colaboraram para a conclusão deste trabalho.

\section{Referências}

AVILA, A. V.; SANCHES, F. M. Correlación de métodos de ajuste para corrección por fallas em sorgo para grano. Agrociência, v. 31, p. 45-64, 1978.

BORÉM, A.; MIRANDA, G. Melhoramento de plantas. 6 ed. Viçosa, MG: Universidade Federal de Viçosa, 2013. $523 \mathrm{p}$.

CANDIDO, L. S.; ANDRADE, J. A. da C.; GARCIA, F. Q.; GONÇALVES, L. S. A.; AMARAL JÚNIOR, A. T. do.
Seleção de progênies de meios-irmãos do composto Isanão VF-1 de milho na safra e safrinha. Ciência Rural, Santa Maria, v. 41, n. 6, p. 947-953, 2011.

DOI: $10.1590 / \mathrm{S} 0103-84782011005000072$.

CARDOSO, M. J.; CARVALHO, H. W. L. de; SANTOS, M. X. dos; LEAL, M. L. S.; OliVeIRA, A. C. de. Desempenho de híbridos de milho na região meio-norte do Brasil. Revista Brasileira de Milho e Sorgo, Sete Lagoas, v. 2, n. 1, p. 43-52, 2003.

DOI: 10.18512/1980-6477/rbms.v2n1p43-52.

CARGNELUTTI FILHO, A.; PERECIN, D.; MALHEIROS, E. B.; GUADAGNIN, J. P. Comparação de métodos de adaptabilidade e estabilidade relacionados à produtividade de grãos de cultivares de milho. Bragantia, Campinas, v. 66, p. 571-578, 2007.

DOI: $10.1590 / \mathrm{S} 0006-87052007000400006$.

CARVALHO, H. W. L.; LEAL, M. L. S.; CARDOSO, M. J.; SANTOS, M. X.; TABOSA, J. N.; SANTOS, M. D.; LIRA, M. A. Adaptabilidade e estabilidade de híbridos de milho em diferentes condições ambientais do nordeste brasileiro. Revista Brasileira de Milho e Sorgo, Sete Lagoas, v. 1, p. 75-82, 2002.

DOI: 10.18512/1980-6477/rbms.v1n2p75-82.

CRUZ, C. D. GENES: a software package for analysis in experimental statistics and quantitative genetics. Acta Scientiarum. Agronomy, Maringá, v. 35, n. 3, p. 271-276, 2013. DOI: 10.4025/actasciagron.v35i3.21251.

CRUZ, C. D.; CASTOLDI, F. L. Decomposição da interação genotípica $\mathrm{x}$ ambientes em partes simples e complexa. Revista Ceres, v. 38, n. 2019, p. 422-430, 1991.

CRUZ, C. D.; REGAZZI, J. A.; CARNEIRO, P. C. S. Modelos biométricos aplicados ao melhoramento genético. 4 ed. Viçosa: Universidade Federal de Viçosa, Viçosa, MG, 2012. v. 1, 514 p.

EBERHART, S. A.; RUSSEL, W. A. Stability parameters for comparing varieties. Crop Science, Madison, v. 6, n. 1, p. 36-40, 1966.

DOI: 10.2135/cropsci1966.0011183X000600010011x. 
GARBUGliO, D. D.; GERAGE, A. C.; ARAÚJO, P. M. de; FONSECA JÚNIOR, N. da S.; SHIOGA, P. S. Análise de fatores e regressão bissegmentada em estudos de estratificação ambiental e adaptabilidade em milho. Pesquisa Agropecuária Brasileira, Brasília, DF, v. 42, n. 2, p. 183191, 2007. DOI: 10.1590/S0100-204X2007000200006.

GONZÁLEZ, A. R. M. Biometria aplicada ao melhoramento do milho amiláceo na região oriental do Paraguai. 2014. 121 f. Dissertação (Mestrado em Genética e Melhoramento) - Universidade Federal de Viçosa, Viçosa, MG, 2014.

LECLERG, E. L. Significance of experimental design in plant breeding. In: FREY, K. J. (Ed.). Plant breeding, a symposium held at Iowa State University. Ames: The Iowa State University Press, 1966. p. 243-313.

LIN, C. S.; BINNS, M. R. A method of analyzing cultivar $\mathrm{x}$ location $\mathrm{x}$ year experiments: a new stability parameter. Theoretical and Applied Genetics, New York, v. 76, n. 3, p. 425-430, 1988.

DOI: $10.1007 / \mathrm{BF} 00265344$.

LÓPEZ, O. E.; GONZÁLEZ, E.; DE LLAMAS, P. A.; MOLINAS, A. S.; FRANCO, E. S.; GARCÍA, S.; RIOS, E. Reconocimiento de suelos, capacidad de uso de la tierra y propuesta de ordenamiento territorial preliminar de la Región Oriental del Paraguay. Asunción: Banco Mundial, 1995. 28 p. Proyecto de Racionalización del uso de la tierra.

MACHADO, V. Productividad y adaptabilidad de cultivares de maíz harinoso Avati Morotî. Investigación Agraria, v. 15, n. 2, p. 75-81, 2013.

MACHADO, V.; NOLDIN, O.; FLORENTIN, M. Habilidad combinatória general y especifica para rendimiento em uma cruza dialélica entre 10 accesiones de maíz amiláceo Avati Morotî. In: REUNIÓN LATINOAMERICANA DE MAÍZ, 19., 2002, Portoviejo, Ecuador. Memórias. Portoviejo: [s.n.], 2002.

PARAGUAI. Dirección de Meteorología e Hidrología de la Dirección Nacional de Aeronáutica Civil. Disponível em: <http://www.meteorologia.gov.py>. Acesso em: 8 mar. 2014.

PIMENTEL-GOMES. Curso de estatística experimental. 13 ed. Piracicaba: Nobel, 1990. 450 p.

RIBEIRO, J. Z.; ALMEIDA, M. I. M. de. Estratificação ambiental pela análise da interação genótipo $\mathrm{x}$ ambiente em milho. Pesquisa Agropecuária Brasileira, Brasília, DF, v. 46, n. 8, p. 875-883, 2011. DOI: 10.1590/S0100-204X2011000800013.

ROBERTSON, A. Experimental design on the measurement of heritabilities and genetic correlations: biometrical genetic. New York: Pergamon Press, 1959.186p.

SCAPIM, C. A.; CARVALHO, C. G.; CRUZ, C. D. Uma proposta de classificação dos coeficientes de variação para cultura de milho. Pesquisa Agropecuária Brasileira, Brasília, DF, v. 30, n. 5, p. 683-686, 1995.

SCOTT, A.; KNOTT, M. Cluster-analysis method for grouping means in analysis of variance. Biometrics, Washington, v. 30, n. 3, p. 507-512, 1974.

SOUZA, F. R. de; RIBEIRO, P. H.; VELOSO, C. A.; CORREA, L. A. Produtividade e estabilidade fenotípica de cultivares de milho em três municípios do Estado do Pará. Pesquisa Agropecuária Brasileira, Brasília, DF, v. 37, n. 9, p. 1269-1274, 2002.

DOI: 10.1590/S0100-204X2002000900010. 